\title{
The influence of free-carrier concentration on the PEC etching of GaN: A calibration with Raman spectroscopy
}

\author{
R. Lewandowska ${ }^{\mathrm{a}, \mathrm{c}}$, J.L. Weyher ${ }^{\mathrm{b}, \mathrm{c}, *}$, J.J. Kelly ${ }^{\mathrm{d}}$, L. Konczewicz ${ }^{\mathrm{a}}$, B. Lucznik ${ }^{\mathrm{c}}$ \\ ${ }^{a}$ Groupe d'Etudes des Semiconducteurs, UM II-CNRS (URA 5650), F 34095 Montpellier cedex 5, France \\ ${ }^{\mathrm{b}}$ Radboud University Nijmegen, IMM, Applied Materials Science, Toernooiveld 1, 6525 ED Nijmegen, The Netherlands \\ ${ }^{\mathrm{c}}$ Institute of High Pressure Physics, Polish Academy of Sciences, ul.Sokolowska 29/37, 01142 Warsaw, Poland \\ ${ }^{\mathrm{d}}$ Debye Institute, University of Utrecht, Princetonplein 1, Utrecht, The Netherlands
}

Received 16 January 2007; received in revised form 4 July 2007; accepted 9 July 2007

Communicated by J.B. Mullin

Available online 14 July 2007

\begin{abstract}
Large areas of electrically active regions of in-homogeneities have been revealed by the electroless photo-etching (PEC) method in GaN layers grown by the hydride vapor phase epitaxy (HVPE) technique.

Variations in the local etch rate have been correlated with the variations in the free-carrier concentrations as determined by microRaman spectroscopy. The etch rate decreased linearly with the log of the carrier concentration. The latter could change by more than two orders of magnitude on the same sample.
\end{abstract}

(C) 2007 Elsevier B.V. All rights reserved.

PACS: 78.30.Fs; 81.05.Ea; 81.65.Cf; 81.70.Fy

Keywords: A1. Defects; A1. Etching; A3. Hydride vapor phase epitaxy; B1. Nitrides

\section{Introduction}

Growth of homo-epitaxial layers of $\mathrm{GaN}$ by hydride vapor phase epitaxy (HVPE) is an attractive solution for obtaining large-area crystals of high structural, electronic and optical quality. However, such layers are not completely free of crystallographic defects and other types of non-homogeneities. Electroless photo-etching (PEC) can reveal not only the crystallographic defects (e.g. dislocations), but also electrically active chemical non-homogeneities (differences in free-carrier concentrations) [1,2]. An advantage of this method is the possibility for either large area or local etching (only the illuminated part of the sample is etched) to map the non-homogeneities on the chosen surface of the sample [3]. However, this method must be calibrated to determine a free-carrier concentra-

\footnotetext{
*Corresponding author. Radboud University Nijmegen, IMM, Applied Materials Science, Toernooiveld 1, 6525 ED Nijmegen, The Netherlands. Tel.: + 31243652 586; fax: + 31243652314 .

E-mail address: J.Weyher@science.ru.nl (J.L. Weyher).
}

tion. In a previous work we found qualitative relationships between the PEC etch rate and Raman spectroscopyderived free-carriers concentrations [2], higher etching depths corresponding to lower free-carriers concentrations. In this paper we report on a quantitative relationship between these two parameters. Such a relationship has been previously established for GaAs etched using DSL (diluted Sirtl-like) photoetching [4,5]. In those works, the authors have shown that the etch rate is proportional to the logarithm of the free-carrier concentration determined using electron beam induced current (EBIC) method. In this communication we used Raman spectroscopy as a tool to determine directly free-carrier concentrations.

\section{Experimental procedure}

Thick HVPE homo-epitaxial GaN layers were grown in a home-made, horizontal HVPE system using a quartz reactor, $\mathrm{N}_{2}$ as the carrier gas and $\mathrm{NH}_{3}$ as the nitrogen source [6]. Typically the epitaxial layers were grown on 
single-crystalline $\mathrm{GaN}$ substrates in [0 0001 l] direction. However, at the edge of the substrates the macroscopic growth directions are $\langle 1 \overline{1} 00\rangle$ and $\langle 11 \overline{2} 0\rangle$. This results in formation of a "frame" on the (0001) surface (which corresponds to so-called wings, as recognized on the (1 $0 \overline{1} 0)$ longitudinal cross-sections in Ref. [2]) with different morphologies and structural and electrical properties.

To eliminate morphological non-homogeneities in the asgrown HVPE layer (large hillocks are typical for the thick layers) the samples were mechanically polished and subsequently subjected to reactive ion etching (RIE) to remove work damage. Wax spots were placed on the sample surface for the purpose of the etch rate measurements. Photoetching in stirred aqueous $\mathrm{KOH}$ solution was performed using illumination from a $450 \mathrm{~W}$ Xe lamp [7,8]. After photoetching of HVPE-grown GaN, apart from the formation of whisker-like features on dislocations, a nano-size "grass" is frequently formed. The nature of the grass has not been established so far despite the attempts made using TEM on the photo-etched samples [9]. To exclude a possible influence of this etching artifact on the free-carrier concentration and depth measurements, we performed our experiments twice. First, the etch depth and Raman measurements were done along lines chosen on the surface of the sample directly after photo-etching. Then the samples were subjected to a newly developed etching procedure (etching in $1 \mathrm{M} \mathrm{KOH}$ solution at $85^{\circ} \mathrm{C}$ for $30 \mathrm{~s}$ [10]) to remove the protruding features from the surface. Finally the surface profile and Raman measurements were repeated.

The etching depth profiles were recorded using an Alfastep profiler. Micro-Raman measurements were performed along the same lines with a Jobin-Yvon T64000 spectrometer at ambient conditions using the $514.532 \mathrm{~nm}$ line of an argon laser as an excitation source. Spectra were taken from the $\left(\begin{array}{lll}0 & 0 & 0\end{array}\right) \mathrm{Ga}$ surfaces in backscattering configuration. The Raman spot was of the order of $1 \mu \mathrm{m}$ in diameter. Surface examination was performed using differential interference contrast (DIC) optical microscopy.

\section{Results and discussion}

\subsection{Determination of the free-carrier concentration using Raman spectroscopy}

In polar crystals, the long-range coulombian interaction splits polar modes $\left(A_{1}\right.$ and $E_{1}$ in the case of $\left.G a N\right)$ into transversal (TO) and (LO) modes. The LO modes can couple with collective vibrations of free carriers-plasmons resulting in modes called longitudinal plasmon-phonon (LPP) modes. From the position of these modes and knowing the position of the uncoupled LO mode, one can find a plasmon frequency $\omega_{\mathrm{p}}$ from the formula (1)

$$
\omega_{ \pm}^{2}=\frac{\left(\omega_{\mathrm{L}}^{2}+\omega_{\mathrm{p}}^{2}-\gamma \Gamma\right) \pm \sqrt{\left(\omega_{\mathrm{L}}^{2}+\omega_{\mathrm{p}}^{2}-\gamma \Gamma\right)^{2}-4 \omega_{\mathrm{p}}^{2} \omega_{\mathrm{T}}^{2}}}{2},
$$

where $\omega_{ \pm}$is the frequency of the high-frequency LPP $(\mathrm{LPP}+)$ mode and the low-frequency LPP (LPP-) mode, respectively; $\omega_{\mathrm{L}}$ and $\omega_{\mathrm{T}}$ are the $\mathrm{LO}$ and TO phonon frequencies, respectively; $\gamma$ is the phonon damping constant and $\Gamma$ is the electron damping constant. The free-carrier concentration $n$ is directly related to the plasmon frequency $\omega_{\mathrm{p}}$ obtained from the LPP modes positions (Eq. (1))

$n=\frac{\omega_{\mathrm{p}}^{2} \varepsilon_{\infty} \varepsilon_{0} m^{*}}{e^{2}}$,

where $\varepsilon_{0}$ is the vacuum permittivity, $m^{*}$ the effective mass of the electron and $e$ is the electron charge. Because we performed our experiments on the surface perpendicular to the $c$-axis of the crystal, only the $\mathrm{A}_{1}$ polar mode was observed. In our calculations we used the following parameters: $\omega\left(\mathrm{A}_{1}(\mathrm{TO})\right)=\omega_{\mathrm{T}}=532 \mathrm{~cm}^{-1}$, $\omega\left(\mathrm{A}_{1}(\mathrm{LO})\right)=\omega_{\mathrm{L}}=736 \mathrm{~cm}^{-1}, \quad \varepsilon_{\infty}=5.35$ and $m^{*}=0.2$ of electron mass [11]. We have neglected damping constants. The uncertainty on the free-carrier concentration values determined was between $5 \%$ (for the higher values) and $20 \%$ (lower values). In this work, the minimum value of the free-carrier concentration which could be determined with a reasonable precision was $10^{17} \mathrm{~cm}^{-3}$. Examples of the Raman spectra of $n-G a N$ are shown in Fig. 1.

\subsection{Etching depth and free-carrier concentration profiles}

Fig. 2 shows a DIC optical image of the GaN surface of one of the investigated samples after photo-etching with the lines $1-1^{\prime}$ and $2-2^{\prime}$ along which the etch depth surface profiles were recorded. Fig. 3 shows a comparison between the etching profile along line $2-2^{\prime}$ and the freecarrier concentration distribution calculated from the $\mathrm{A}_{1}(\mathrm{LO})$ mode frequency. The profiles of the free-carrier concentration bear a very strong resemblance to the respective profiles of the etch depth (similar results were obtained on three different samples and several linescans), confirming a previously observed qualitative correlation between these values [2]. Subsequently, the etch rates were calculated and the relationship with the corresponding values of the free-carrier concentrations was established. For the investigated range $\left(1 \times 10^{17}-5 \times\right.$ $10^{18} \mathrm{~cm}^{-3}$ ) a linear relationship was found between the logarithm of the free-carrier concentration $n\left(\mathrm{in} \mathrm{cm}^{-3}\right)$ and the etch rate $R$ (in $\mathrm{nm} / \mathrm{min}$ ). The results are presented in Fig. 4. A linear function was fit to the experimental results for each profile. This relationship is of the form

$R=\left(-\frac{1}{\beta}\right) \ln \left(\frac{n}{n_{\mathrm{th}}}\right)$,

where $R$ is the etch rate, $n$ is the free-carrier concentration, $n_{\text {th }}$ is the free-carrier concentration corresponding to $R=0$ (it is specific to a given sample and can be obtained by extrapolation of the $R=f(n)$ lines) and $\beta$ is a slope coefficient. As shown in Fig. 4, the slopes of the $R=f(n)$ lines are very similar within the limits of experimental 


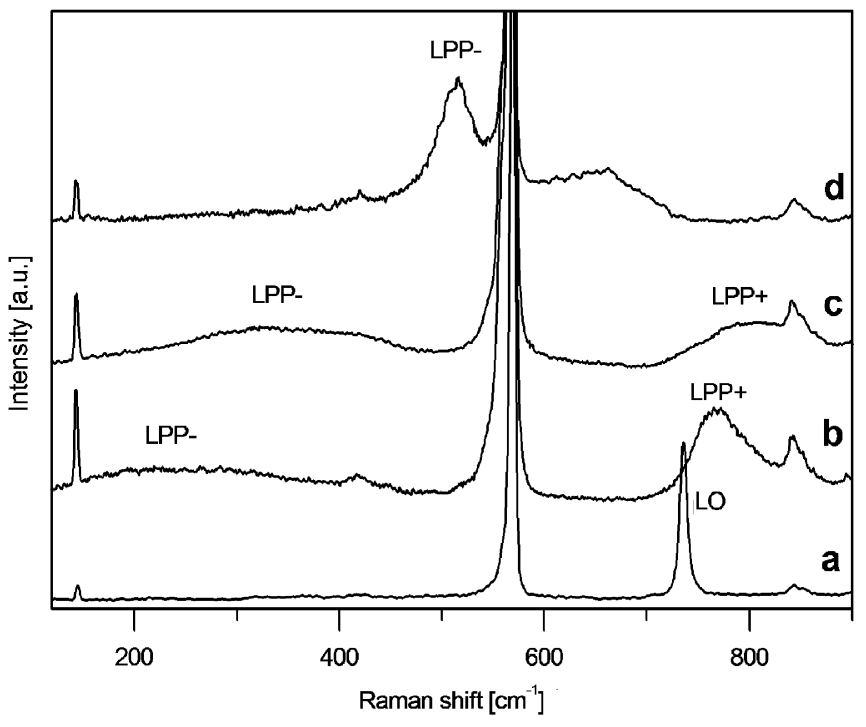

Fig. 1. Raman spectra of n-GaN with different doping levels; (a) LO mode is not shifted and no LPP modes are observed, it means that there is no phonon-plasmon coupling, because the free-carrier concentration is too low and can be estimated as $<10^{17} \mathrm{~cm}^{-3}$; (b) both LPP + (at $770 \mathrm{~cm}^{-1}$ ) and LPP - (at $220 \mathrm{~cm}^{-1}$ ) modes are observed, calculated freecarrier concentration is $1.25 \times 10^{18} \pm 0.05 \mathrm{~cm}^{-3}$; (c) LPP $+\left(\right.$ at $803 \mathrm{~cm}^{-1}$ ) and $\mathrm{LPP}-\left(\right.$ at $\left.326 \mathrm{~cm}^{-1}\right)$ are observed, free-carrier concentration is $2.9 \pm 0.1 \times 10^{18} \mathrm{~cm}^{-3} ;$ (d) only LPP- mode $\left(\right.$ at $515 \mathrm{~cm}^{-1}$ ) is shown, LPP + was found at $1887 \mathrm{~cm}^{-1}$, calculated free-carrier concentration is $4 \times 10^{19} \mathrm{~cm}^{-3}$

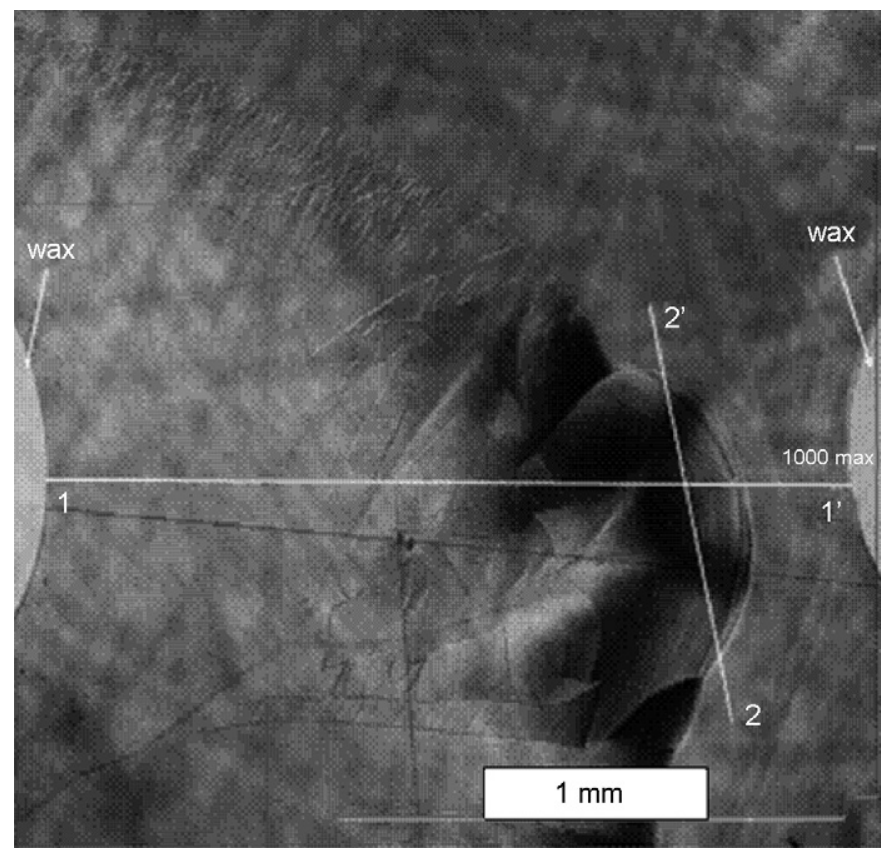

Fig. 2. DIC optical image of the GaN surface after photo-etching with the lines $1-1^{\prime}$ and $2-2^{\prime}$ along which the etch depth surface profiles were recorded.

error. Lines from profiles $1-1^{\prime}$ and $2-2^{\prime}$ of sample 1 overlap as would be expected for measurements performed on the same sample (the same $n_{\text {th }}$ ) but the results from sample 2

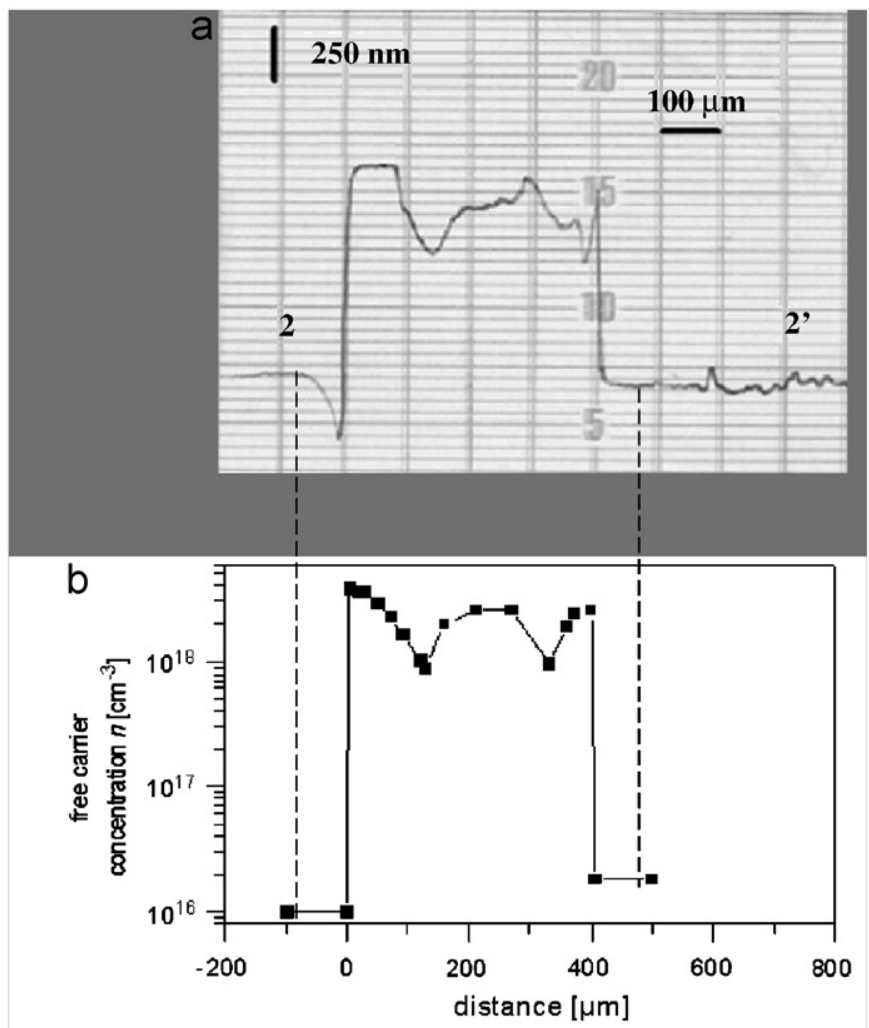

Fig. 3. (a) Comparison of the etch depth profile along the line 2-2' (Fig. 2) performed using a profilometer and (b) free-carrier concentration profile calculated from the position of the LPP + modes.

are shifted $\left(n_{\text {th }}\right.$ values are different for samples grown separately under different conditions, e.g. $\left(n_{\mathrm{th}}\right)_{1-1^{\prime}}=$ $\left(n_{\mathrm{th}}\right)_{2-2^{\prime}}=6 \times 10^{18} \mathrm{~cm}^{-3}$ and $\left(n_{\mathrm{th}}\right)_{3-3^{\prime}}=7 \times 10^{17} \mathrm{~cm}^{-3}$ in Fig. 4). Sample regions with a carrier concentration higher than $n_{\text {th }}$ will not be etched. Indeed, on all three samples there were parts of the surface showing no noticeable etching depth (e.g. the "frames" and also some areas in the central part of the samples). The carrier concentrations of these areas are represented by the encircled points lying on the abscissa in Fig. 4 and clearly they do not comply with the linear relationship $R=f(n)$. The explanation of these seemingly puzzling experimental results can be given on the basis of the principles of electroless etching established for GaAs [12] and $\mathrm{GaN}$ [13]. Fig. 5 shows a schematic representation of the photocurrent-potential curves for a sample containing areas of different carrier concentrations in a broad range $\left(n-n^{++}\right)$. The onset of the photocurrent $j$ shifts to higher potential $V$ as the carrier concentration increases $\left(n \rightarrow n^{++}\right)$[13]. The rest (or mixed) potential of the system, determined by oxygen reduction at the counter electrode [13], is indicated by $V_{\mathrm{m}}$. From Fig. 5 it is clear that at this potential the photocurrent, and thus the photoetch rate, decreases as $n$ increases $\left(j_{\mathrm{m}}^{n}>j_{\mathrm{m}}^{n+}\right)$ while above $n_{\text {th }}$ no etching is observed. The value of $n_{\mathrm{th}}$ clearly depends on the particular sample. 


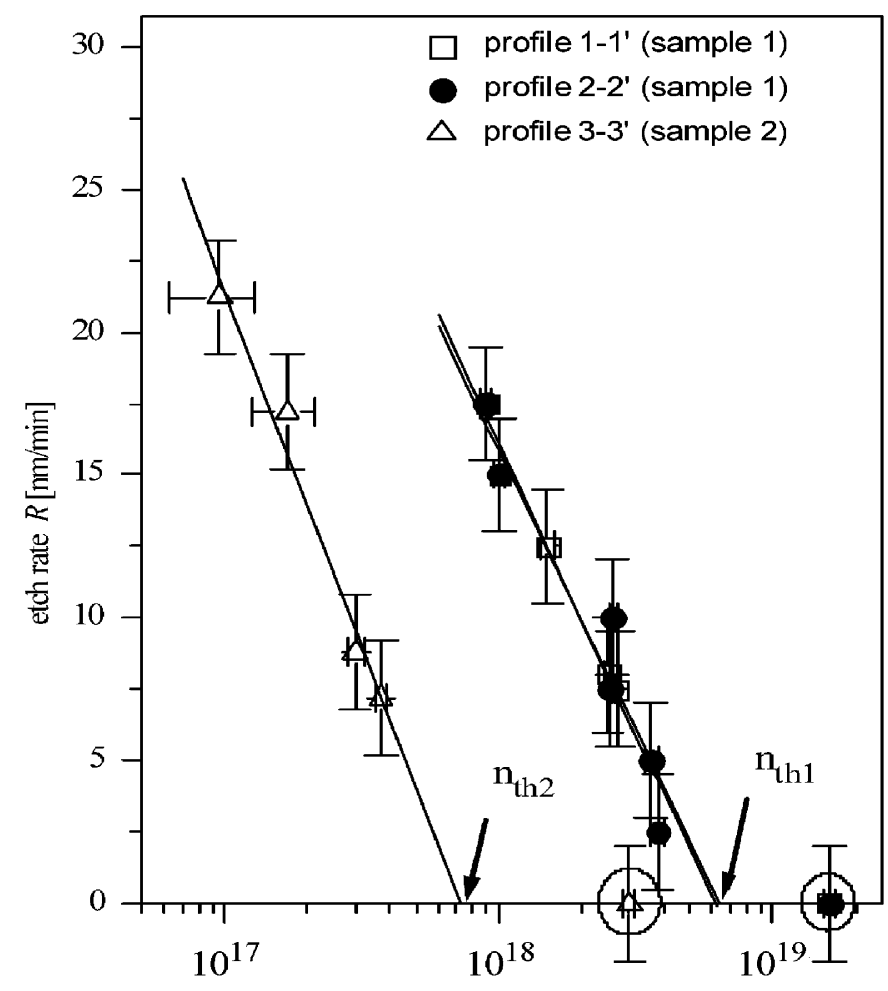

free carrier concentration $n\left[\mathrm{~cm}^{-3}\right]$

Fig. 4. Relationships between the logarithm of the free-carrier concentration $n$ (determined using Raman spectroscopy) and the etch rate $R$ (PEC etching method). The equations of the linear fitting are as follows: $R_{1-1^{\prime}}=$ $-20 \log _{10}(n)+370 ; \quad R_{2-2^{\prime}}=-20 \log _{10}(n)+380 ; \quad R_{3-3^{\prime}}=-25 \log _{10}(n)+450$. For the encircled points, see text.

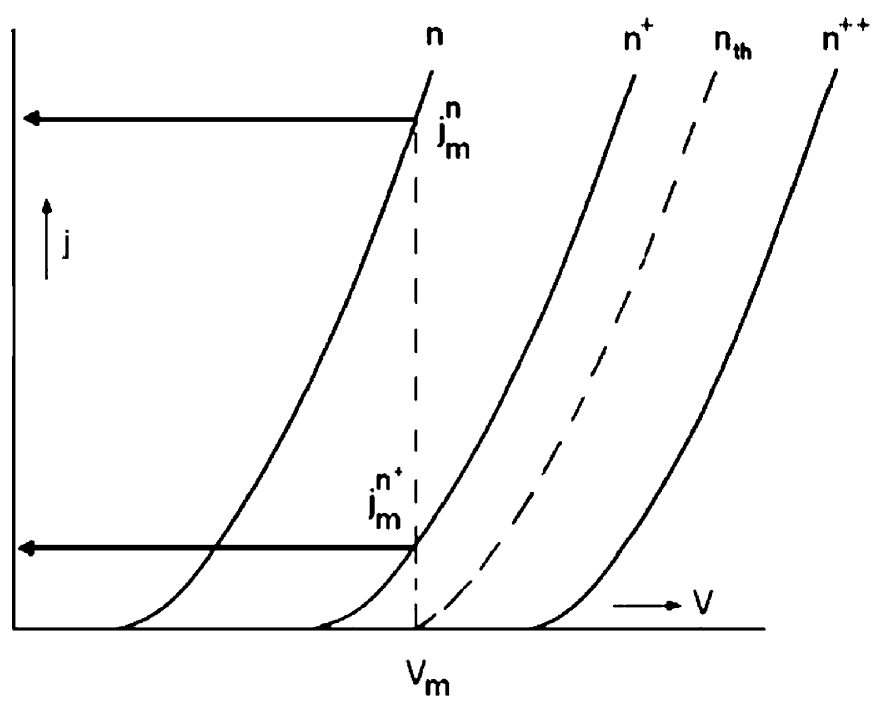

Fig. 5. Simplified photocurrent-potential plots to demonstrate etch rates in n-type $\mathrm{GaN}$ containing areas of markedly different carrier concentrations. Only the low photocurrent range is shown. $j_{\mathrm{m}}^{n}$ and $j_{\mathrm{m}}^{n+}$ are partial currents arising at areas of $n$ and $n+$ carrier concentration, respectively. $V_{\mathrm{m}}$ is the rest (or mixed) potential of the sample [13].
We also compared the results obtained before and after removing the "grass" and it was found that the etch depth changed by less than $10 \%$, with no marked influence on the results of the calibration (for the profile $1-1^{\prime}$ analyzed without removing of the "grass", we found $R=-21 \log _{10}(n)+400$ was found $)$.

\section{Conclusions}

Large differences in etch rate and in carrier concentration were found by surface profiling and by local Raman measurements on thick homo-epitaxial GaN layers grown by the HVPE method. A linear relationship between the etch rate of $\mathrm{GaN}$ and the logarithm of the free-carrier concentration in the $1 \times 10^{17}-5 \times 10^{18} \mathrm{~cm}^{-3}$ range was found, in agreement with the results obtained on GaAs. Electrochemical concepts can be used to explain the co-existence in one n-type sample of areas of very large etch rate differences including areas of zero etch rate. These results constitute the basis for the rapid evaluation of carrier concentration differences across a photoetched GaN sample from simple etch depth (etch rate) measurements.

\section{Acknowledgments}

We acknowledge the financial support of the Netherlands Foundation for Technical Research (STW) as well as CNRS (France) and PAN (Poland) in the frame of the Associate European Laboratory NodLAB. Critical remarks of Dr. C. Frigeri are kindly acknowledged.

\section{References}

[1] J.L. Weyher, L. Macht, F.D. Tichelaar, H.W. Zandbergen, P.R. Hageman, P.K. Larsen, Mater. Sci. Eng. B 280 (2002) 91.

[2] J.L. Weyher, R. Lewandowska, L. Macht, B. Lucznik, I. Grzegory, Mater. Sci. Semicond. Process. 9 (2006) 175.

[3] J.L. Weyher, L. Macht, Eur. Phys. J. Appl. Phys. 27 (2004) 37.

[4] C. Frigeri, J.L. Weyher, L. Zanotti, J. Electrochem. Soc. 136 (1989) 262.

[5] C. Frigeri, J.L. Weyher, L. Zanotti, Mat. Res. Soc. Symp. Proc., Pittsburg 138 (1989) 527;

C. Frigeri, J.L. Weyher, L. Zanotti, Mater. Res. Soc., Pittsburg 138 (1989) 527.

[6] B. Łucznik, et al., J. Crystal Growth 281 (2005) 38.

[7] C. Youtsey, I. Adesida, G. Bulman, Appl. Phys. Lett. 71 (1997) 2151.

[8] J.L. Weyher, F.D. Tichelaar, H.W. Zandbergen, L. Macht, P.R. Hageman, J. Appl. Phys. 90 (2001) 6105.

[9] Z. Liliental-Weber, F. Tichelaar, J.L. Weyher, unpublished results.

[10] J.J. Kelly, L. Macht, D.H. van Dorp, M.R. Kooijman, J.L. Weyher, Electrochem. Soc. Proc. (2005-04) 138.

[11] A.S. Barker Jr., M. Ilegems, Phys. Rev. B 7 (1973) 743.

[12] J. van de Ven, J.L. Weyher, J.E.A.M. van den Meerakker, J.J. Kelly, J. Electrochem. Soc. 133 (1986) 799.

[13] L. Macht, J.J. Kelly, J.L. Weyher, A. Grzegorczyk, P.K. Larsen, J. Crystal Growth 273 (2005) 347. 